Ciencias Agrarias/Agricultural Sciences

Cienc Tecn UTEQ (2017) 10(2) p 75-82 ISSN 1390-4051; e-ISSN 1390-4043

(c) (1) (1)(

DOI: https://doi.org/10.18779/cyt.v10i2.170

\title{
Uso de la distribución normal para cuantificar la calidad del riego en sistemas de aspersión estacionario
}

\section{Use of normal distribution to quantify irrigation quality in stationary sprinkler irrigation systems}

\author{
${ }^{\bullet}$ Jose Nicolás Ortiz Romero ${ }^{1}$ \\ ${ }^{1}$ Universidad Centroccidental Lisandro Alvarado (UCLA), Decanato de Agronomía, Departamento de Ingeniería Agrícola. \\ Cabudare-Lara, Apartado postal 400.Venezuela. ¡jortiz@ucla.edu.ve
}

Rec.: 12.05.2017. Acept.: 16.09.2017.

Publicado el 1 de diciembre de 2017

\section{Resumen}

E: el campo experimental de riego del Decanato de Agronomía de la UCLA se llevó a cabo un estudio cuyo objetivo fue cuantificar el reparto del agua y los parámetros de calidad del riego en un sistema de aspersión estacionario durante dos ciclos del cultivo de caraota usando la distribución normal. Se determinó el área adecuadamente regada (AAR) en 13 eventos de riego, y esta se relacionó con los parámetros eficiencia de aplicación (Efa), pérdidas por percolación, eficiencia de almacenamiento (Efal) y el déficit. Los resultados mostraron que la Efal y la percolación aumentaron con el incremento del AAR; mientras, la Efa y el déficit disminuyeron con el aumento del AAR. Los parámetros de calidad del riego en función del AAR se ajustaron apropiadamente a una regresión lineal, donde los coeficientes y el modelo resultaron significativos $(\mathrm{p}<0.001)$ y el error estándar de la estimación fue bajo.

Palabras clave: área adecuadamente regada, eficiencia de aplicación, percolación.
Tn the experimental irrigation field of the Agronomy Faculty of UCLA (University Centroccidental Lisandro Alvarado, Venezuela), a study whose aim was quantify the water distribution and irrigation quality parameters was carried out using the normal distribution in a stationary sprinkler irrigation system during two crops or cycles of black bean (Phaseolus vulgaris L.). Through 13 irrigation events, the Area Adequately Watered (AAW) was determined and it related to the application efficiency parameters (Efa), percolation losses, storage efficiency (Efal) and deficit (or water shortage). Results showed that Efal and percolation losses increased with rising AAW; while the Efa and the deficit decreased with the increase of the AAW. Irrigation quality parameters as a function of AAW were appropriately adjusted to a linear regression, where coefficients and model were significant ( $p$ $<0.001$ ) and the standard error of estimation was low.

Key words: area adequately watered, application efficiency, percolation. 
Introducción

L a aplicación del agua en riego por aspersión, aun cuando exista un buen solapamiento entre los diámetros de mojado de los aspersores, no es totalmente uniforme en el área regada; es decir, que unas zonas reciben la cantidad de agua que requiere el cultivo y otras mayores o menores aportes de lo necesario. Jahad (2013), señaló que, con una cuidadosa selección del tamaño de boquillas, presión de funcionamiento y espaciamiento entre aspersores, la cantidad de agua necesaria a reponer en la zona radical del cultivo se puede hacer con buena uniformidad y adecuada a la velocidad de infiltración del suelo.

Por otra parte, cuando se aplica un riego no toda el agua queda almacenada en el perfil del suelo donde se encuentran las raíces, si no que una porción puede perderse por evaporación, escorrentía y percolación profunda. El resultado de un riego es que, como media, se infiltre una lámina y una proporción del área "a" recibe al menos la lámina de agua que se pretendía aportar, quedando el resto (1-a) con déficit. El regante puede decidir que fracción de área "a" quiere que quede bien regada, no resultando económicamente rentable, en la generalidad de los casos, que toda la parcela reciba al menos la dosis neta (Montero, 1999). Tradicionalmente, para el estudio de la uniformidad se ha utilizado la expresión de Christiansen (1942), la cual está basada en una representación estadística de las láminas de agua colectadas en un determinado marco de riego. El coeficiente de uniformidad no debe ser el parámetro que determine si el área está bien regada o no, sino el porcentaje de área regada adecuadamente (Jiménez et al., 2012).

La distribución poblacional de una variable cuantitativa se puede describir por su media $(\mu)$ y su desviación típica $(\sigma)$, y también mediante una curva de densidad, que representa las frecuencias relativas como áreas bajo la curva. El tipo más importante de curva de densidad es la distribución normal. Las láminas de agua aplicadas a un área regada por 4 aspersores se adecuan a una distribución normal, y según Hart y Reinolds (1965) esto es válido para un amplio rango de tamaño de aspersores (5 a 300 GPM) cuando estos son razonablemente operados y espaciados. La densidad de probabilidad acumulada de la distribución normal, mostrada como una curva-S, indica la frecuencia o la probabilidad que valores menores o mayores que un valor específico ocurran. Para una lámina de riego dada, la curva-S, puede ser usada para calcular el porcentaje de área adecuadamente regada y los parámetros de calidad del riego (Anyoji y Wu, 1994). Cuando la lámina media aplicada es igual al requerimiento neto del cultivo, el 50\% del área será subirrigada; mientras, el restante $50 \%$ queda sobreregada o adecuadamente regada (Merkley, 2001). La curva-S fue usada por Hart y Reinolds (1965) para determinar la eficiencia de aplicación en riego por aspersión. El objetivo del trabajo fue cuantificar el reparto del agua y los parámetros de calidad del riego en sistemas de aspersión estacionario utilizando la distribución normal.

\section{Materiales y métodos}

\section{Ubicación y características de la zona}

El ensayo se realizó en el Campo Experimental de Riego del Decanato de Agronomía de la UCLA en el núcleo Tarabana, Cabudare, estado Lara, Venezuela, ubicado geográficamente a $10^{\circ} 01^{\prime} 52^{\prime}$ " de latitud norte y $69^{\circ} 16^{\prime} 44^{\prime \prime}$ de longitud oeste, y 510 msnm. Los datos climáticos medios anuales de la zona son: temperatura de $25{ }^{\circ} \mathrm{C}$, precipitación de $813 \mathrm{~mm}$, humedad relativa de $75 \%$, radiación solar de $371{\mathrm{cal} . \mathrm{cm}^{-}{ }^{2} \mathrm{y}}^{2}$ una evaporación de $2085 \mathrm{~mm}$.

\section{Control del agua aplicada}

Se estableció el cultivo de caraota durante dos ciclos, se aplicaron 13 riegos en el primero y 9 en el segundo, evaluándose únicamente 13 de los 22 riegos. Los mismos se administraron con una frecuencia semanal luego de la germinación, emergencia y arraigo del cultivo. La evapotranspiración de referencia (ETo) se estimó por el método de la tina clase "A" (Doorenbos y Pruitt, 1977). Los datos de evaporación se obtuvieron de una tina ubicada en el mismo campo experimental que presentaba un coeficiente de tina $(\mathrm{Kp})$ de 0.79 , determinado experimentalmente para la zona por Pire y Rodríguez (2007). Para el cálculo de la evapotranspiración del cultivo (ETc) se usaron los coeficientes de cultivo (Kc) tabulados por la FAO (Allen et al., 1998).

Para el riego se utilizó un sistema de aspersión fijo temporal, formado por una subunidad de riego que constaba de una tubería principal y 4 tuberías laterales separadas $10 \mathrm{~m}$, con cuatro aspersores en cada línea. Los aspersores se colocaron a una distancia de $10 \mathrm{~m}$, y a una altura de $1 \mathrm{~m}$ sobre el suelo. Del área total del ensayo (40 x $40 \mathrm{~m}$ ), se seleccionó el área delimitada por los 4 aspersores centrales $\left(100 \mathrm{~m}^{2}\right)$ que se dividió en 25 sub-parcelas de 2 x 2 m y en el centro de cada una se colocó un pluviómetro (de $10 \mathrm{~cm}$ de diámetro por $15 \mathrm{~cm}$ de alto). Se usaron aspersores modelo Rain Bird $14 \mathrm{~J} 囚$ provistos de una boquilla de $2.38 \mathrm{~mm}$ de diámetro, operando a una presión de 40 PSI. La Figura 1 presenta la disposición de los 25 pluviómetros en el área de ensayo. Aunque los pluviómetros utilizados no tienen la medida estándar recomendada para medir el volumen de agua captada, Playan et al. (2005) evaluaron el efecto del tamaño del pluviómetro $(7.9,13$ y $24 \mathrm{~cm}$ de diámetro) en la determinación de las pérdidas por evaporación y arrastre, encontraron que, para valores de vientos moderados, inferiores $3 \mathrm{~m} \mathrm{~s}^{-1}$, las diferencias no resultaban importantes.

\section{Distribución del agua aplicada en los riegos}

Para determinar la distribución del agua se utilizó la metodología propuesta por Anyoji y Wu (1994). Con el apoyo de la Figura 2, para una lámina de riego requerida, especificada como $\mu+\alpha \sigma$, se forman cuatro áreas (A, B, C y D). Estas cuatro áreas representan la cantidad total de agua aplicada sobre un área base y su proporción relativa. La cantidad almacenada 

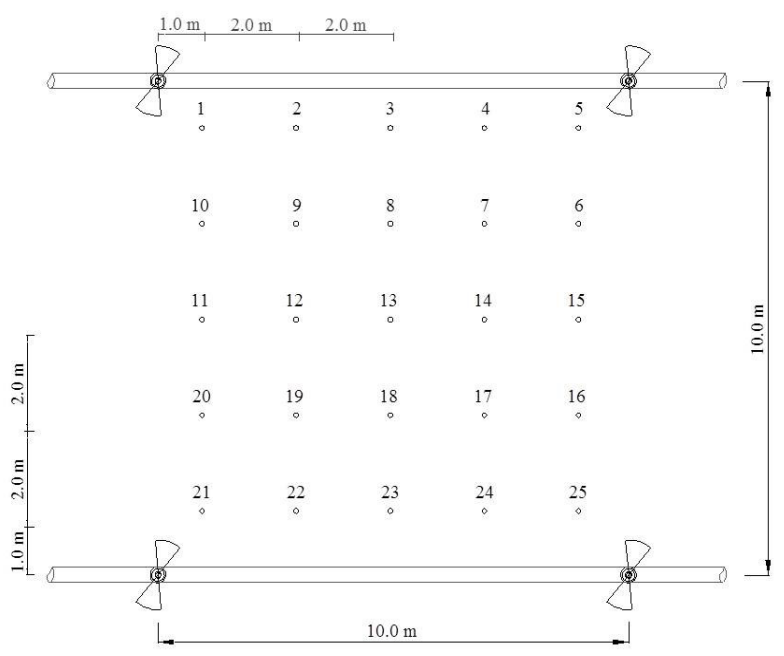

Figura 1. Distribución de los pluviómetros en el área control

en la zona radical $(\mathrm{A}+\mathrm{C})$, la percolación profunda $(\mathrm{B})$ y la cantidad de déficit (D) pueden ser evaluadas basada en la distribución normal.

Basada en la distribución normal (Figura 3), la densidad de probabilidad acumulada para una población más grande que $\mu+\alpha \sigma$ se expresa como:

$\mathrm{a}=\int_{\mu+\alpha \sigma}^{\infty} f(y) d y$ y $\mathrm{f}(\mathrm{y})$ la función de probabilidad de la distribución normal, $\mathrm{y}$ puede expresarse como:

$$
f(y)=\frac{1}{\sigma \sqrt{2 \pi}} e^{-\frac{1}{2}\left(\frac{y-\mu}{\sigma}\right)^{2}}
$$

donde " $y$ " es una variable en la población y "e" es la constante transcendental. Considerando $\mathrm{u}=(\mathrm{y}-\mu) / \sigma)$, se llega a la ecuación (3):

donde "a" representa la densidad de probabilidad acumulada

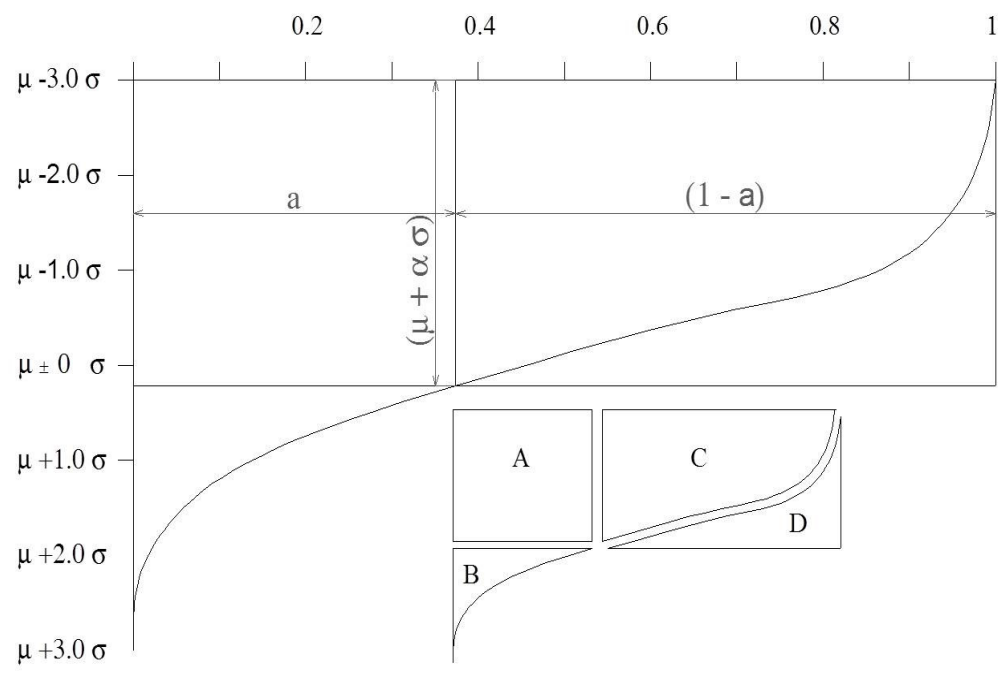

Figura 2. Densidad de probabilidad acumulada de la distribución normal $(\mu+\alpha \sigma$ es la lámina de riego requerida) 


$$
f(u)=\frac{1}{\sigma \sqrt{2 \pi}} e^{-\frac{u^{2}}{2}}
$$

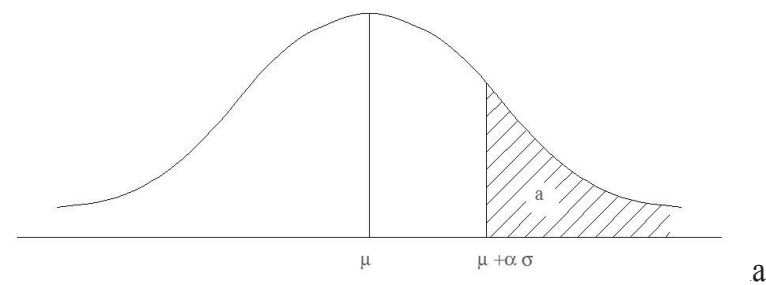

distribución normal

Integrando la ecuación (1) resulta:

$\mathrm{a}=\int_{\alpha}^{\infty} \frac{1}{\sqrt{2 \pi}} e^{-\frac{u^{2}}{2}} d u$

El valor de "a", como una función de $\alpha$, se puede obtener por integración o a través de tablas estadísticas para una distribución normal. La densidad de probabilidad acumulada para la población mayor que $\mu+\alpha \sigma$ representa el área adecuadamente regada y la población menor que $\mu+\alpha \sigma$ es 1-a. Si la variable $\mu+\alpha \sigma$ es considerada como la lámina de riego requerida (Figura 2), el área $(\mathrm{A}+\mathrm{B})$ puede ser evaluada como:

$A+B=\int_{\mu+\alpha \sigma}^{\infty} y * f(y) d y$

que puede ser resuelta como:

$A+B=\frac{\sigma}{\sqrt{2 \pi}} e^{\frac{\alpha^{2}}{2}}+\mu a$

El área "A" puede determinarse simplemente como Figura 2:

$A=a(\mu+\alpha \sigma)$

El área "B" se puede determinar restando la ecuación 7 de la 6

$B=\frac{\sigma}{\sqrt{2 \pi}} e^{-\frac{\alpha^{2}}{2}}-\mathrm{a} \alpha \sigma$

La cantidad total de agua de riego aplicada sobre un área base se puede expresar como el área $(\mathrm{A}+\mathrm{B}+\mathrm{C})$ :

$A+B+C=\mu$

el área $(\mathrm{A}+\mathrm{C})$ se puede obtener restando la ecuación 8 de la 9:

$A+C=\mu+\mathrm{a} \alpha \sigma-\frac{\sigma}{\sqrt{2 \pi}} e^{-\frac{\alpha^{2}}{2}}$

También, restando la ecuación 7 de la 10, el área “C” se puede determinar como:

$C=\mu(1-\mathrm{a})-\frac{\sigma}{\sqrt{2 \pi}} e^{-\frac{\alpha^{2}}{2}}$

Desde la figura 2, el área $(\mathrm{C}+\mathrm{D})$ se puede calcular simplemente como:

$C+D=(1-\mathrm{a})(\mu+\alpha \sigma)$

El área "D" se determina restando la ecuación 11 de la 12:

$D=(1-\mathrm{a}) \alpha \sigma+\frac{\sigma}{\sqrt{2 \pi}} e^{-\frac{\alpha^{2}}{2}}$

Para calcular la probabilidad de la variable normal, con media $\mu$ y desviación típica $\sigma, \mathrm{N}(\mu, \sigma)$, se transformó a una variable normal tipificada, $\mathrm{N}(0,1)$. La correspondencia entre la escala tipificada (z) y la escala " $y$ " se puede expresar mediante la fórmula:

$z=\frac{y-\mu}{\sigma}$

donde $y$ representa la lámina de agua requerida por el cultivo (ETc) en un momento dado, $\mu$ la lámina de agua media (recogida en los 25 pluviómetros colocados en el área de estudio, (Figura 1) del riego que se aplica para suplir la lámina requerida y $\sigma$ la desviación típica de las láminas captada en los pluviómetros.

Para un determinado valor de $\mathrm{z}$, en tablas estadísticas para una distribución normal o a través del programa Excel (distribución normal estandarizada del valor z), se puede obtener el área que recibió láminas de agua inferiores a la requerida por el cultivo; finalmente, para obtener el área adecuadamente regada (AAR), se resta el área anterior de 1.

\section{Parámetros de calidad del riego}

La eficiencia de aplicación (Efa) se calculó en base a la cantidad de agua que llega al suelo (la recogida en los pluviómetros), que coincide con la lámina infiltrada, pues, la escorrentía fue nula al ser la pluviometría del aspersor menor a la infiltración básica del suelo, por tanto, la única pérdida de agua fue por percolación profunda, y es un complemento de la Efa, esta se cuantificó como (Figura 2):

$$
\text { Ef } a=\frac{A+C}{A+B+C} * 100
$$

Las pérdidas por percolación (PP), eficiencia de almacenamiento (Efal) y el porcentaje de déficit (PD) se calcularon con las ecuaciones 16,17 y 18 , respectivamente:

$$
\begin{aligned}
& P P=\frac{B}{A+B+C} * 100 \\
& E f a l=\frac{A+C}{A+C+D} * 100
\end{aligned}
$$


$P D=\frac{D}{A+B+C} * 100$

Se utilizó análisis de regresión para relacionar la lámina de agua recogida en los pluviómetros, la eficiencia de aplicación, la percolación, la eficiencia de almacenamiento y el deficif con el área adecuadamente regada. Para ello se usó el programa informático Statgraphics v 5.1 (Statistical Graphics Corp., Englewood Cliffs, USA).

\section{Resultados y discusión}

\section{Distribución del agua aplicada}

En el Cuadro 1, se presenta la distribución del agua en el perfil del suelo de los riegos evaluado en los dos ciclos del cultivo, de acuerdo al área adecuadamente regada (AAR) se obtiene la proporción relativa del agua almacenada en la zona radical $(A+C)$, la percolación profunda (B) y el déficit (D). Se observa como varia el AAR en función de la lámina media de agua recogida (LAR) y del requerimiento de agua del cultivo (ETc), siendo mayor el AAR cuando LAR es superior al ETc, creciendo el AAR cuando la diferencia entre LAR y ETc es mayor. Si se aplicara justo lo que el cultivo requiere, el $50 \%$ del área quedaría adecuadamente regada y el otro $50 \%$ subirrigada; por ello, cuando la LAR fue mayor al ETc el AAR supera el $50 \%$, y fue inferior al $50 \%$ en caso contrario, lo que está en concordancia a lo afirmado por Merkley (2001). Analizando el agua almacenada en el suelo, es de resaltar que cuando aumenta el AAR o área sobre irrigada, los porcentajes de la fracción A y B, con relación a la LAR, se incrementan; mientras, el porcentaje de la fracción C disminuye; así, cuando el AAR pasa de 35 a $85 \%$, la proporción A pasa de 36.63 a $71.27 \%$ y B pasa de 2.70 a $17.06 \%$, en tanto, $\mathrm{C}$ disminuye de 60.59 a $11.67 \%$.

En la Figura 4, se muestra la distribución del agua para un riego en particular (Riego 8) cuando las necesidades hídricas del cultivo (ETc) fueron $18.46 \mathrm{~mm}$, en la misma se observa que, aproximadamente, $83 \%$ del área recibió una lámina de agua superior a la ETc. La lámina promedio aplicada en el riego fue $21.66 \mathrm{~mm}$, quedando almacenado en la zona radical $18.02 \mathrm{~mm}(\mathrm{~A}+\mathrm{C})$, la lámina percolada (B) fue $3.64 \mathrm{~mm}$ y el déficit (D) $0.44 \mathrm{~mm}$. En el riego resultó una Efa de 83.18\%, percolación de $16.82 \%$, Efal de $97.60 \%$ y $2.05 \%$ de déficit. Queda en evidencia que el uso de esta metodología permite conocer cómo se distribuye la cantidad de agua aplicada en cada riego; así como, cuantificar los parámetros de calidad del riego, en contraste con la metodología de Christiansen (1942) que sólo permite calcular el coeficiente de uniformidad del agua, parámetro que sólo da una idea de cuán grande es el déficit de riego respecto al promedio aplicado; por tanto, no es un buen indicador de si un área queda bien regada o no, lo que coincide con lo afirmado por Jiménez et al. (2012). En la figura se observa que las láminas muestreadas en el marco de riego (LAR) en función a la fracción del área se ajustaron muy bien a un polinomio de tercer orden, donde los coeficientes y el modelo resultaron significativos $(\mathrm{P}<0.001)$; además, de un valor bajo del error estándar de la estimación.

Cuadro 1. Distribución del agua aplicada en base a ETc y lamina media de agua recogida

\begin{tabular}{cccccccc}
\hline NR & ETc & LAR & AAR & A & B & C & D \\
\hline 1 & 10.61 & 12.37 & 81.00 & 8.59 & 1.97 & 1.81 & 0.21 \\
2 & 15.63 & 14.97 & 35.00 & 5.49 & 0.41 & 9.07 & 1.07 \\
3 & 25.39 & 24.65 & 43.00 & 11.03 & 1.44 & 12.18 & 2.18 \\
4 & 36.27 & 37.42 & 58.00 & 21.12 & 2.83 & 13.47 & 1.68 \\
5 & 19.19 & 22.80 & 85.00 & 16.25 & 3.89 & 2.66 & 0.28 \\
6 & 33.95 & 32.31 & 40.00 & 13.44 & 1.75 & 17.12 & 3.39 \\
7 & 29.69 & 30.56 & 61.00 & 17.98 & 1.78 & 10.80 & 0.91 \\
8 & 18.46 & 21.66 & 80.00 & 14.69 & 3.64 & 3.33 & 0.44 \\
9 & 13.86 & 14.61 & 61.00 & 8.40 & 1.52 & 4.68 & 0.77 \\
10 & 37.38 & 36.88 & 46.00 & 17.16 & 1.70 & 18.03 & 2.20 \\
11 & 36.71 & 34.89 & 39.00 & 14.25 & 1.75 & 18.90 & 3.57 \\
12 & 37.54 & 35.85 & 38.00 & 14.29 & 1.48 & 20.08 & 3.17 \\
13 & 21.77 & 22.71 & 56.00 & 12.10 & 3.16 & 7.45 & 2.22 \\
\hline
\end{tabular}

NR: Número de riego; ETc: Evapotranspiración del cultivo (mm); LAR: Lámina aplicada media recogida en los pluviómetros (mm); AAR: Área adecuadamente regada (\%); A + C: Lámina almacenada en la zona radical (mm); B: Percolación (mm); D: Déficit (mm). 
Ortiz, 2017

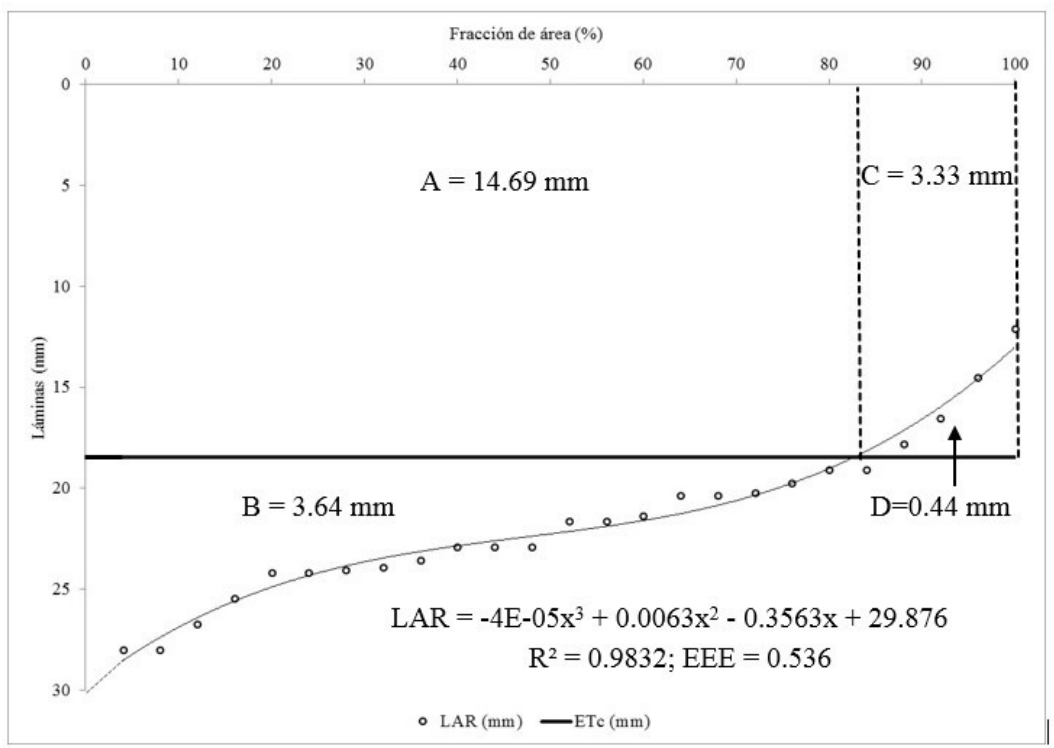

Figura 4. Distribución del agua aplicada en un riego en particular

\section{Parámetros de calidad del riego}

La Figura 5, muestra la relación de la eficiencia de aplicación (Efa) y de las pérdidas por percolación profunda (PP) en función al área adecuadamente regada (AAR), se observa, para un AAR de 35\% se obtiene una Efa de 97\% y una PP del 3\%. Al aumentar el AAR disminuye la Efa y se incrementan las PP; se consigue un punto de equilibrio donde se interceptan las líneas de Efa y PP, con valores de 85 y $15 \%$, respectivamente. Cuando el AAR es $80 \%$, significa que es el porcentaje de área que debe recibir una lámina de riego mayor o igual a la requerida por el cultivo para asegurar una eficiencia de aplicación aceptable y menos lavado de productos químicos hacia estratos más profundos que la zona radical. Trabajos pioneros en esta misma línea realizados por Hart y Reynolds (1965) indican que el 80\% es un valor apropiado de área adecuadamente regada. Igualmente, Montero (1999) señaló que, generalmente, no resulta económicamente rentable, que toda la parcela reciba al menos la dosis neta, sino un porcentaje del área regada. Tanto, la Efa como las PP se ajustaron adecuadamente a un modelo de regresión lineal, lo cual se confirmó por la alta significancia de los coeficientes y del modelo $(\mathrm{P}<0.001)$, y por el bajo error estándar de la estimación.

En cuanto a la eficiencia de almacenamiento y al déficit, los valores en todos los riegos evaluados en los dos ciclos, fueron superiores a $90 \%$ e inferiores a $11 \%$, respectivamente; valores que son próximos a los encontrados por Anyoji y $\mathrm{Wu}$ (1994), quienes reportan cifras por encima del 92\%

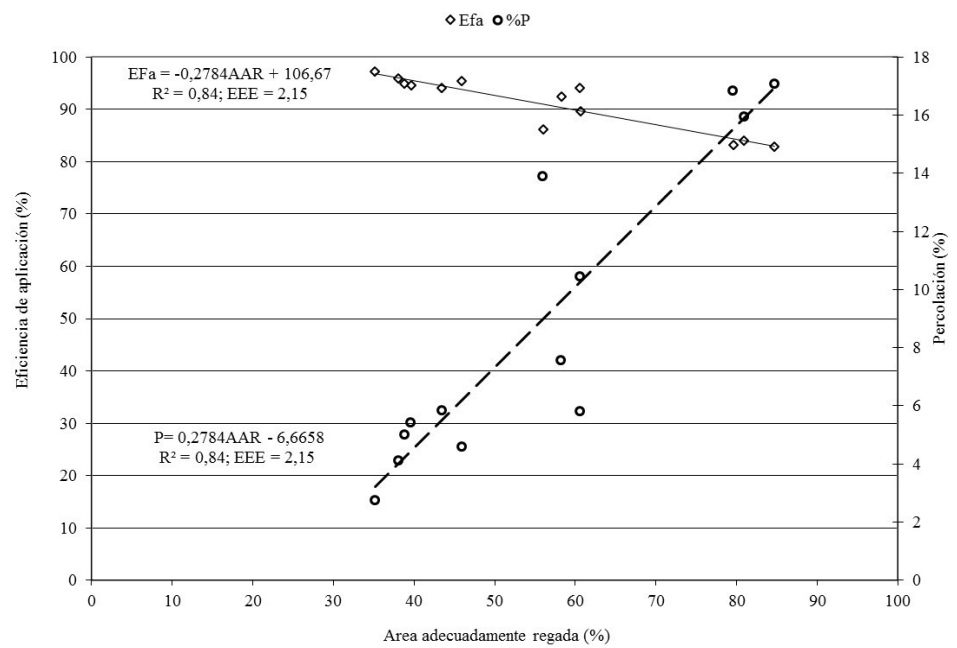

Figura 5. Eficiencia de aplicación (Efa) y percolación (P) en función del área adecuadamente regada (AAR) 


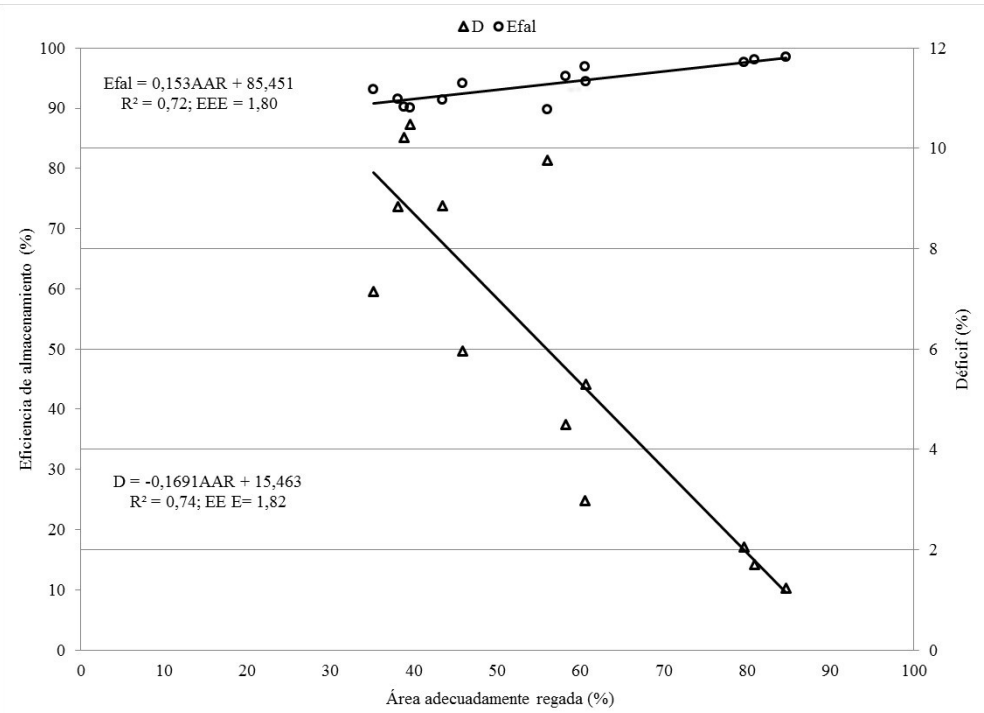

Figura 6. Eficiencia de almacenamiento (Efal) y déficit (D) en función del área adecuadamente regada (AAR)

y menores de $8 \%$ para la Efal y déficit, respectivamente, trabajando en riego por goteo, método que, generalmente, aplica el agua más uniforme que el riego por aspersión. En la figura 6, se presenta la evolución de la relación de la eficiencia de almacenamiento (Efal) y del déficit en función al área adecuadamente regada (AAR), en la misma se observa que la Efal aumenta en la medida que el AAR es mayor; contrariamente, el déficit disminuye cuando se incrementa el AAR. Ambos parámetros de calidad del riego se ajustaron a una regresión lineal, donde el intercepto, el termino constante y el modelo resultaron significativos $(p<0.001)$ e igualmente presentaron un error estándar de la estimación bajo, lo que confirma la adecuada bondad del modelo.

\section{Conclusiones}

T a metodología utilizada permitió cuantificar la distribución del agua en el perfil del suelo y los parámetros de calidad del riego (Área adecuadamente regada, eficiencia de aplicación, percolación, eficiencia de almacenamiento y déficit), en contraste con la metodología de Christiansen que sólo proporciona el coeficiente de uniformidad.

Cuando el área adecuadamente regada (AAR) pasa de 35 a $85 \%$, la cantidad de agua almacenada en la zona radical (A) pasa de 37 a $61 \%$ y la percolación (B) de 3 a $17 \%$, en tanto, la cantidad subirrigada (C) disminuye de 61 a $12 \%$.

La eficiencia de aplicación fue 97 y $83 \%$ para un AAR de 35 y $85 \%$, respectivamente; mientras que la eficiencia de almacenamiento paso de 93 a 99\% y el déficit disminuyo de 7 a 1\%. Los parámetros de calidad del riego en función del AAR se ajustaron apropiadamente a una regresión lineal.

\section{Bibliografía}

Allen, R., Pereira, L., Raes, D., and Smith, M. (1998). Crop evapotranspiration. Guidelines for computing crop water requirements. FAO irrigation and drainage, paper $\mathrm{n}^{0} 56, \mathrm{FAO}$, Rome.

Anyoji, H., and Wu, I. (1994). Normal distribution water application for drip irrigation schedules. Transactions of the ASAE. 37(1): 159-164.

Christiansen, J. (1942). Irrigation by sprinkling. University of California. Agricultural Experiment Station. Bulletin 670. $124 \mathrm{pp}$.

Doorenbos, J., and Pruitt, W. (1977). Las necesidades de agua de los cultivos. Estudio FAO Riego y Drenaje $n^{\circ}$ 24. FAO, Roma.

Hart, W., and Reynolds, W. (1965). Analytical desing of sprinkler systems. Transactions of the ASAE. 8(1): 8385.

Jahad, U. (2013). Study the emission uinformity coefficients for sprinkler irrigation system with different layouts. Euphrates Journal of Agriculture Science. 5 (4): 1-10.

Jiménez, E., González, P. y Domínguez, M. (2012). Relación entre parámetros de uniformidad de riego en máquinas de pivote central. Rev. Cie. Téc. Agr. 21(3): 18-22.

Merkley, G. (2001). Design Efficiency for Sprinkler Irrigation Systems. Lecture Notes. Utah State Uni $\neg$ versity, Logan. USA.

Montero, J. (1999). Análisis de la distribución de agua en sistemas de riego por aspersión estacionario. Desarrollo del modelo de simulación de riego por aspersión SIRIAS. Tesis Doctoral. ETSIA, Universidad de Castilla-La Mancha. Albacete-España. 
Pire, R. y Rodríguez R. (2007). Evapotranspiración referencial (ETo) medida vs estimada en Tarabana ( $10^{\circ} 01^{\prime}$ LN), estado Lara, Venezuela. Revista Unellez Ciencias Técnicas. 25: 58-66.

Playan, E., Salvador, R., Faci, J., Zapata N., Martinez-Cob A., and Sánchez, I. (2005). Day and night wind drift and evaporation losses in sprinklers solid-set and moving lateral. Agricultural Water Management. 76: 139-159. 